\title{
Crystal structure of 8-methyl-8-phenyl-[8H]-pyrano[3,2-f]quinoline, $\mathrm{C}_{19} \mathrm{H}_{15} \mathrm{NO}$
}

\author{
S. Aldoshin, I. Chuev, O. Filipenko
}

Institut of Chemical Physics in Chernogolovka, 142432, Chernogolovka, Moscow region, Russia

\section{Lokshin, R. Guglielmetti}

LCMOM. ERS 158, Faculté des Sciences de Luminy, Case 901, 13288 Marseille Cedex 9, France

and G. Pèpe

Universités d'Aix-Marseille II et III, Centre de Recherche sur les Mécanismes de la Croissance Cristalline, Campus de Luminy, Case 913 ,

13288 Marseille Cedex 9, France

Received March 25, 1998, CSD-No. 409285

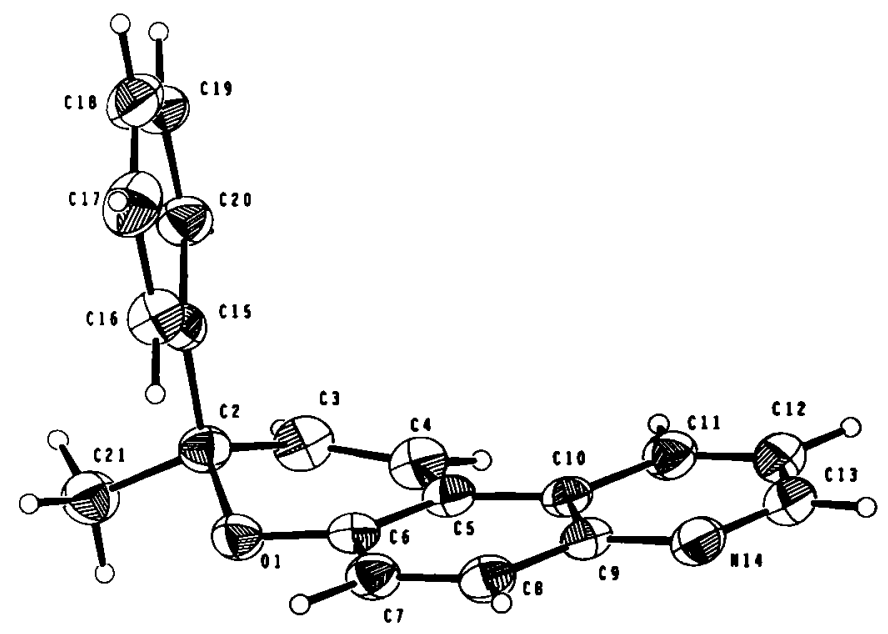

Source of material: The compound $\mathrm{C}_{19} \mathrm{H}_{15} \mathrm{NO}$ was obtained by condensation of $\beta$-methylcinnamaldehyde with 6-hydroxyquinoline in presence of tetraethyl orthotitanate using toluene as solvent (see ref. 1). Crystals were obtained from heptane solution.

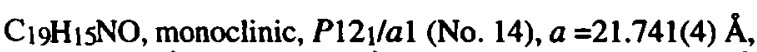
$b=7.584(2) \AA, c=8.802(2) \AA, \beta=97.30(3)^{\circ}, V=1439.5 \AA^{3}, Z=4$, $R(F)=0.038, R_{\mathrm{w}}\left(F^{2}\right)=0.116$.
Table 1. Parameters used for the $\mathrm{X}$-ray data collection

\begin{tabular}{|c|c|}
\hline $\begin{array}{l}\text { Crystal: } \\
\text { Wavelength: }\end{array}$ & $\begin{array}{l}\text { colorless needle, size } 0.10 \times 0.16 \times 0.43 \mathrm{~mm} \\
\text { Mo } K_{\alpha} \text { radiation }(0.71073 \AA)\end{array}$ \\
\hline$\mu:$ & $0.78 \mathrm{~cm}^{-1}$ \\
\hline Diffractometer: & $\mathbf{K M}-4$ \\
\hline Scan mode: & $\boldsymbol{\theta}$ \\
\hline $\mathbf{T}_{\text {measuremens: }}$ & $\begin{array}{l}293 \mathrm{~K} \\
54.04^{\circ}\end{array}$ \\
\hline $\mathbf{N}(h k)_{\text {unique: }}$ & 2937 \\
\hline Criterion for $I_{0}$ : & $I_{0}>2 \sigma\left(I_{0}\right)$ \\
\hline $\mathrm{N}(\text { param })_{\text {refined. }}$ & 251 \\
\hline Programs: & SHEL XS-86, SHELXI-93 \\
\hline
\end{tabular}

Table 2. Final atomic coordinates and displacement parameters (in $\AA^{2}$ )

\begin{tabular}{llllll}
\hline Atom & Site & $x$ & $y$ & $z$ & $U_{\text {iso }}$ \\
\hline $\mathbf{H}(3)$ & $4 e$ & $0.3322(8)$ & $1.004(3)$ & $-0.052(2)$ & $0.077(5)$ \\
$\mathbf{H}(4)$ & $4 e$ & $0.4132(9)$ & $1.150(3)$ & $0.092(2)$ & $0.082(6)$ \\
$\mathbf{H}(7)$ & $4 e$ & $0.5211(7)$ & $0.522(3)$ & $0.149(2)$ & $0.070(5)$ \\
$\mathbf{H}(8)$ & $4 e$ & $0.5995(8)$ & $0.645(2)$ & $0.339(2)$ & $0.066(4)$ \\
$\mathbf{H}(11)$ & $4 e$ & $0.4740(9)$ & $1.252(3)$ & $0.294(2)$ & $0.078(5)$ \\
$\mathbf{H}(12)$ & $4 e$ & $0.5550(9)$ & $1.349(3)$ & $0.483(2)$ & $0.087(6)$ \\
$\mathbf{H}(13)$ & $4 e$ & $0.6374(9)$ & $1.155(2)$ & $0.566(2)$ & $0.072(5)$ \\
$\mathbf{H}(16)$ & $4 e$ & $0.390(1)$ & $0.460(3)$ & $0.176(2)$ & $0.099(6)$ \\
$\mathbf{H}(17)$ & $4 e$ & $0.336(1)$ & $0.342(4)$ & $0.376(3)$ & $0.121(8)$ \\
$\mathbf{H}(18)$ & $4 e$ & $0.247(1)$ & $0.480(3)$ & $0.441(3)$ & $0.108(7)$ \\
$\mathbf{H}(19)$ & $4 e$ & $0.207(1)$ & $0.726(3)$ & $0.319(2)$ & $0.102(7)$ \\
$\mathbf{H}(20)$ & $4 e$ & $0.2624(9)$ & $0.858(3)$ & $0.124(2)$ & $0.082(5)$ \\
$\mathbf{H}(211)$ & $4 e$ & $0.2895(9)$ & $0.702(2)$ & $-0.161(2)$ & $0.076(5)$ \\
$\mathbf{H}(212)$ & $4 e$ & $0.352(1)$ & $0.718(3)$ & $-0.231(3)$ & $0.095(6)$ \\
$\mathbf{H}(213)$ & $4 e$ & $0.3356(9)$ & $0.534(3)$ & $-0.141(2)$ & $0.091(6)$ \\
\hline
\end{tabular}

Table 3. Final atomic coordinates and displacement parameters (in $\AA^{2}$ )

\begin{tabular}{lllllllllll}
\hline Atom & Site & $x$ & $y$ & $z$ & $U_{11}$ & $U_{22}$ & $U_{33}$ & $U_{12}$ & $U_{13}$ & $U_{23}$ \\
\hline $\mathrm{O}(1)$ & $4 e$ & $0.42625(4)$ & $0.6764(1)$ & $0.0192(1)$ & $0.0464(5)$ & $0.0644(6)$ & $0.0590(6)$ & $0.0063(4)$ & $0.0119(4)$ & $-0.0089(4)$ \\
$\mathrm{C}(2)$ & $4 e$ & $0.36316(6)$ & $0.7433(2)$ & $0.0062(2)$ & $0.0448(6)$ & $0.0625(8)$ & $0.0539(8)$ & $0.0072(5)$ & $0.0077(5)$ & $0.0034(6)$ \\
$\mathrm{C}(3)$ & $4 e$ & $0.36507(7)$ & $0.9417(2)$ & $0.0097(2)$ & $0.0565(8)$ & $0.0636(9)$ & $0.069(1)$ & $0.0113(6)$ & $0.0050(7)$ & $0.0148(7)$ \\
$\mathrm{C}(4)$ & $4 e$ & $0.41203(7)$ & $1.0262(2)$ & $0.0881(2)$ & $0.0601(8)$ & $0.0500(7)$ & $0.0689(9)$ & $0.0077(6)$ & $0.0134(6)$ & $0.0131(6)$ \\
$\mathrm{C}(5)$ & $4 e$ & $0.46403(5)$ & $0.9280(2)$ & $\mathbf{0 . 1 6 7 4 ( 2 )}$ & $\mathbf{0 . 0 4 8 0 ( 6 )}$ & $\mathbf{0 . 0 4 7 7 ( 7 )}$ & $\mathbf{0 . 0 5 3 0 ( 8 )}$ & $\mathbf{0 . 0 0 2 9 ( 5 )}$ & $0.0174(5)$ & $0.0076(5)$ \\
$\mathrm{C}(6)$ & $4 e$ & $0.46889(6)$ & $0.7533(2)$ & $0.1265(2)$ & $0.0435(6)$ & $0.0541(7)$ & $0.0498(7)$ & $0.0033(5)$ & $0.0155(5)$ & $0.0005(5)$ \\
\hline
\end{tabular}


Table 3. (Continued)

\begin{tabular}{|c|c|c|c|c|c|c|c|c|c|c|}
\hline Atom & Site & $x$ & $y$ & $z$ & $U_{11}$ & $U_{22}$ & $U_{33}$ & $U_{12}$ & $U_{13}$ & $U_{23}$ \\
\hline$C(7)$ & $4 e$ & $0.51975(6)$ & $0.6489(2)$ & $0.1857(2)$ & $0.0484(7)$ & $0.0525(7)$ & $0.0655(9)$ & $0.0086(5)$ & $0.0165(6)$ & $-0.0016(6)$ \\
\hline$C(8)$ & $4 e$ & $0.56491(6)$ & $0.7185(2)$ & $0.2893(2)$ & $0.0452(6)$ & $0.0608(8)$ & $0.0661(9)$ & $0.0083(6)$ & $0.0123(6)$ & $0.0041(6)$ \\
\hline $\mathrm{C}(9)$ & $4 e$ & $0.56167(6)$ & $0.8946(2)$ & $0.3391(2)$ & $0.0448(6)$ & $0.0607(7)$ & $0.0531(8)$ & $-0.0022(5)$ & $0.0165(5)$ & $0.0038(6)$ \\
\hline$C(10)$ & $4 e$ & $0.51063(6)$ & $1.0006(2)$ & $0.2773(2)$ & $0.0481(6)$ & $0.0498(7)$ & $0.0546(8)$ & $-0.0028(5)$ & $0.0213(5)$ & $0.0049(5)$ \\
\hline $\mathrm{C}(11)$ & $4 e$ & $0.50907(7)$ & $1.1746(2)$ & $0.3339(2)$ & $0.0620(8)$ & $0.0499(7)$ & $0.072(1)$ & $-0.0037(6)$ & $0.0222(7)$ & $0.0031(6)$ \\
\hline$C(12)$ & $4 e$ & $0.55550(8)$ & $1.2317(2)$ & $0.4408(2)$ & $0.072(1)$ & $0.0632(9)$ & $0.075(1)$ & $-0.0169(7)$ & $0.0271(8)$ & $-0.0102(8)$ \\
\hline$C(13)$ & $4 e$ & $0.60389(8)$ & $1.1179(3)$ & $0.4936(2)$ & $0.0618(9)$ & $0.083(1)$ & $0.062(1)$ & $-0.0201(8)$ & $0.0182(7)$ & $-0.0081(8)$ \\
\hline$N(14)$ & $4 e$ & $0.60802(5)$ & $0.9544(2)$ & $0.4461(1)$ & $0.0507(6)$ & $0.0793(9)$ & $0.0613(8)$ & $-0.0079(6)$ & $0.0132(5)$ & $-0.0002(6)$ \\
\hline$C(15)$ & $4 e$ & $0.33104(6)$ & $0.6671(2)$ & $0.1359(2)$ & $0.0446(6)$ & $0.0558(7)$ & $0.0489(7)$ & $0.0020(5)$ & $0.0047(5)$ & $-0.0034(5)$ \\
\hline$C(16)$ & $4 e$ & $0.35160(8)$ & $0.5145(2)$ & $0.2114(2)$ & $0.0630(8)$ & $0.0618(8)$ & $0.072(1)$ & $0.0047(7)$ & $0.0104(7)$ & $0.0087(7)$ \\
\hline$C(17)$ & $4 e$ & $0.3205(1)$ & $0.4444(3)$ & $0.3260(2)$ & $0.087(1)$ & $0.083(1)$ & $0.076(1)$ & $-0.017(1)$ & $0.0023(9)$ & $0.021(1)$ \\
\hline$C(18)$ & $4 e$ & $0.26858(9)$ & $0.5247(3)$ & $0.3649(2)$ & $0.071(1)$ & $0.117(2)$ & $0.064(1)$ & $-0.033(1)$ & $0.0124(8)$ & $0.003(1)$ \\
\hline$C(19)$ & $4 e$ & $0.24718(8)$ & $0.6751(3)$ & $0.2901(2)$ & $0.0536(8)$ & $0.113(2)$ & $0.075(1)$ & $-0.0098(9)$ & $0.0207(7)$ & $-0.016(1)$ \\
\hline$C(20)$ & $4 e$ & $0.27806(7)$ & $0.7460(2)$ & $0.1769(2)$ & $0.0478(7)$ & $0.077(1)$ & $0.069(1)$ & $0.0071(7)$ & $0.0111(6)$ & $-0.0039(8)$ \\
\hline$C(21)$ & $4 e$ & $0.33242(9)$ & $0.6721(3)$ & $-0.1465(2)$ & $0.0626(9)$ & $0.102(1)$ & $0.053(1)$ & $-0.0032(9)$ & $0.0057(7)$ & $-0.0010(9)$ \\
\hline
\end{tabular}

Acknowledgment. This work was supported by Russian Foundation of Basic Research (grant N 0398/96).

\section{References}

1. Harié, G.: Synthése, Etude du Comportement Photochronique et de la Barrière d'ouverture Themique du Cycle Pyrane de $2 H$-Cromènes et Spiro-2H-Chromènes Carbocycliques. Thesis, Université de la Méditerranée Marseille, France 1996.
2. Jonhson, C. K: ORTEP II. Report ORNL- 3974, Oak Ridge National Laboratory, Tennessee, USA 1976.

3. Sheldrick, G. M.: SHELX-86. Program for the Determination of Crystal Structures. University of Göttingen, Germany 1985.

4. Sheldrick, G. M.: SHELXL-93. Program for the Refinement of Crystal Structures. University of Göttingen, Germany 1993. 